\title{
Assessing North American Forest Disturbance from the Landsat Archive
}

\author{
Jeffrey G. Masek, Robert Wolfe, Forrest Hall \\ Hydrospheric and Biospheric Sciences Laboratory \\ NASA Goddard Space Flight Center \\ Greenbelt, MD 20771, USA \\ Jeffrey.G.Masek@nasa.gov \\ Warren Cohen, Robert Kennedy, Scott Powell \\ PNW Research Laboratory \\ USDA Forest Service \\ Corvallis, OR 97331, USA
}

\author{
Samuel Goward, Chengquan Huang \\ Department of Geography \\ University of Maryland \\ College Park, MD 20742, USA
}

\author{
Sean Healey, Gretchen Moisen \\ Rocky Mountain Research Station \\ USDA Forest Service \\ Ogden, UT 84401, USA
}

\begin{abstract}
Forest disturbances are thought to play a major role in controlling land-atmosphere fluxes of carbon. Under the auspices of the North American Carbon Program, the LEDAPS (Landsat Ecosystem Disturbance Adaptive Processing System) and NACP-FIA projects have been analyzing the Landsat satellite record to assess rates of forest disturbance across North America. In the LEDAPS project, wall-to-wall Landsat imagery for the period 1975-2000 has been converted to surface reflectance and analyzed for decadal losses (disturbance) or gains (regrowth) in biomass using a spectral "disturbance index". The NACP-FIA project relies on a geographic sample of dense Landsat image time series, allowing both disturbance rates and recovery trends to be characterized. Preliminary results for the 1990's indicate high rates of harvest within the southeastern US, Eastern Canada, and the Pacific Northwest, with spatially averaged $(\sim 50 \times 50 \mathrm{~km})$ turnover periods as low as 25-40 years. Lower rates of disturbance are found in the Rockies and Northeastern US.
\end{abstract}

Keywords-disturbance, Landsat, forests, atmospheric correction, change detection, biomass

\section{INTRODUCTION}

Forest disturbance and recovery have been regarded as critical, but poorly quantified, mechanisms for transferring carbon between the land surface and the atmosphere. [1, 2]. Disturbance events (fires, harvest, storm damage, insect infestation) tend to emit carbon to the atmosphere through oxidation and decomposition of wood. On the other hand, recovery from past disturbance tends to sequester carbon from the atmosphere since young forests tend to be highly productive and have lower levels of heterotrophic respiration [3]. The balance of these processes across the landscape is one control on overall (net) ecosystem productivity.

The North American Carbon Program (NACP) is an interagency research activity designed to quantify sources and sinks of carbon across North America. Given the importance of forest disturbance within the overall carbon cycle, the NACP has called for improved information on the timing and extent of forest disturbance from historical satellite data to support improved modeling of carbon dynamics.

Two ongoing projects sponsored by NASA are characterizing recent North American forest disturbance by mining the Landsat archive. The first project (Landsat Ecosystem Disturbance Adaptive Processing System or LEDAPS) is using a decadal, wall-to-wall change detection to map disturbance rates from 1990-2000. The second project, NACP-FIA, is using a stratified random sample of 25 Landsat image time series to construct detailed disturbance histories. In this paper we discuss each project in turn, and present initial results.

\section{LEDAPS PROJECT}

\section{A. Objectives}

The overall goal of the LEDAPS disturbance mapping is to provide wall-to-wall mapping of stand-clearing forest disturbance across North America for the period 1990-2000. To do this, we are relying on the decadal Landsat GeoCover data set produced by Earth Satellite Corporation (now MDA Federal) through NASA's Science Data Purchase program. The GeoCover dataset is a global collection of orthorectified, mostly cloud-free Landsat imagery, centered on 1975, 1990, and 2000 epochs [4]. Actual image acquisition dates vary depending on data availability, but for North America most acquisitions date from 1986-1992 for the 1990-epoch coverage, and 1999-2001 for the 2000-epoch coverage. In addition, not all images were acquired during peak-greenness conditions, and the 1990 and 2000 epoch data were not necessarily acquired during the same month.

The mapping effort is targeted toward evaluating standclearing disturbance (biomass loss primarily from clear cuts 
and fires) and recovery (biomass gain). However, given the 10 -year observation interval, it is not possible to distinguish between permanent forest cover conversion and disturbance, nor is it always possible to distinguish older stand clearing events from more recent non-clearing disturbance.

\section{B. LEDAPS Processing Approach}

Landsat TM and ETM+ imagery are first calibrated to top-of-atmosphere reflectance, and then atmospherically corrected using the MODIS/6S methodology [5]. The resulting surface reflectance products form the basis for the change detection algorithm that follows.

To map forest disturbance, we use the tasseled-cap Disturbance Index [6]. The Disturbance Index (DI) records the normalized spectral distance of any given pixel from a nominal "mature forest" class to a "bare soil" class. The DI is calculated using the Tasseled Cap (brightness-greennesswetness) indices for Landsat TM/ETM+ reflectance factor:

$$
D I=B^{\prime}-\left(G^{\prime}+W^{\prime}\right)
$$

where B', G', and W' represent the Tasseled Cap brightness, greenness, and wetness indices normalized by the mean and standard deviation of the mature forest class for each Landsat scene. In LEDAPS, we use the MODIS Vegetation Continuous Fields (VCF) treecover product to provide a basis for identifying mature forest stands and normalizing the tasseled cap indices. We then calculate the decadal change in DI (DDI) between two Geocover images large increases generally correspond to disturbance events; large decreases correspond to regrowth. The final step of the algorithm is to filter out changes in DI that are not related to forest dynamics (e.g. cropping patterns, shrubland phenology, etc) using an internal forest/non-forest classifier.

Both the atmospheric correction and disturbance mapping reuse the software architecture of the MODIS MODAPS processing system. In essence, we are trying to leverage the advances in mass processing developed for the NASA Earth Observing System for Landsat applications. Individual science algorithms are coded as PGE (product generation executables) and use HDF-EOS as input/output format. Jobs are scheduled and run on a cluster of Intel processors running Linux. The current North American 1990-2000 data set includes $\sim 2200$ images. Reprocessing the data set takes about two weeks with the current LEDAPS hardware configuration.

\section{Validation}

Annual/biennial image time series from the NACP-FIA project (described below are being used to produce detailed records of disturbance through semi-automated techniques (classification followed by visual inspection and hand editing). Three $15 \times 15 \mathrm{~km}$ blocks are extracted from each Landsat path-row used by the NACP-FIA project. These disturbance histories are then compared to the LEDAPS results at three scales: pixel-level, $1 \mathrm{~km}$ scale, and $15 \mathrm{~km}$ (whole block) scale.

Our analysis to date suggests that the LEDAPS analyses show relatively low errors of commission $(20-30 \%$ of true disturbance amount), but higher errors of omission (30-50\% of the true disturbance amount). This reflects the limitations of the 10-year imaging interval between the 1990 and 2000 Geocover products. Detection accuracy of older disturbance events (ie. 1990-1994) is low, since regrowth on those plots by the 2000 acquisition masks the change signal. Nevertheless, by establishing empirical relations between our LEDAPS estimates and the true disturbance rates, we can adjust the LEDAPS estimates to compensate for the "missing" disturbance signal.

\section{Initial LEDAPS Results}

High rates of disturbance due to timber harvest are found throughout the southeastern United States, Maine/Quebec, and the Pacific Northwest. In these regions, turnover rates of up to $2 \%$ per year are common. Fire scars are apparent across the Boral zone of Canada, consistent with a fire return interval on the order of 100 years. Disturbance rates in other regions (e.g. mid-Atlantic, Ohio Valley, southern Rockies) are lower. In part this is due to limitations in the 10-year mapping interval - selective harvest of hardwoods is difficult to detect in the LEDAPS analysis. However, forest age maps from the US Forest Service do show generally older forests in these regions, indicating lower rates of harvest.

In most regions, clearing and regrowth appear to be roughly balanced during the 1990's. However, there are local exceptions. In northern Maine and eastern Texas, harvesting appears to have spread into relatively undisturbed secondary forests during the period. In the Olympic Peninsula, the cessation of harvest on state forests has led to a preponderance of young regrowth.

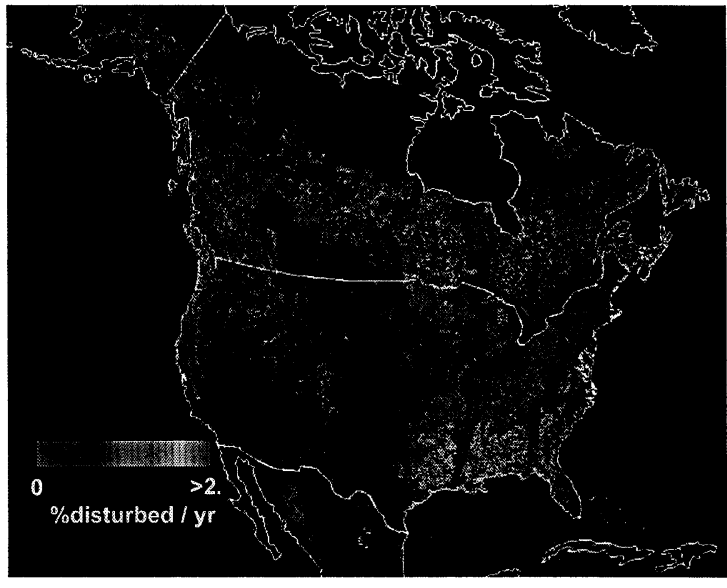

Figure 1. LEDAPS disturbance map for North America. Disturbance rates for each $500 \times 500 \mathrm{~m}$ cell are calculated as the percent of cell area disturbed during the measurement interval (nominally 1990-2000). Color scale ranges from 0 (purple/black) to over $2 \%$ per year (red) 


\section{NACP-FIA PROJECT}

\section{A. Overview}

The NACP-FIA project led by Dr. Samuel Goward (University of Maryland) takes a different, but complementary approach to mapping disturbance within the United States. Twenty-five Landsat path/row locations were selected via stratified random sample to represent the major forest types within the United States. For each of these locations, a set of annual or biennial Landsat images was assembled, primarily from the latter half of the local growing season, for the period 1972-2004. This dense time series allows the history of forest disturbance and variability in recovery to be assessed for each pixel in the data sets. Furthermore, by linking pixel-scale reflectance to plot measurements by the US Forest Service FIA (Forest Inventory and Analysis) program, changes in pixel reflectance can be related directly to changes in biomass.

\section{B. $\quad$ Sampling Strategy}

The challenges in acquiring, processing and analyzing imagery and FIA data in each scene are considerable, making wall-to-wall coverage impractical with with a 1-2 year image acquisition interval. Therefore, a sampling approach was developed for national-level estimates of forest dynamics, with each Landsat scene a single sample unit. The sampling approach needed to fulfill several competing goals, including capturing diversity of forest types, minimizing inclusion of scenes with little forest cover, geographic dispersion of scenes, and inclusion of certain targeted "focal" scenes.

To meet these goals, we developed an unequalprobability sampling approach. The unique area of WRS-2 scenes were tessellated using Thiessaen Scene Areas (TSAs) [7] which were then used as the sampling grid. For scenes with greater than $2 \%$ cumulative forest cover, 100,000 randomized ordered lists (ROTLs) of TSAs were created, and for each potential sample, we calculated critera scores based on scene dispersion, forest diversity, forest cover area, and presence of focal scenes. The ROTLs were then ranked according to each criterion score, and one ROTL was selected from the subset that maximized all criteria. Finally, probabilities of inclusion were calculated for each scene as the proportion of ROTLs in the final set in which that scene occurs. These probabilities allow for unequal-probability, design-based estimation.

\section{Disturbance Analysis}

Each image in the 25 time series "data cubes" is first orthorectified using the Geocover 2000 as geodetic control and the SRTM $90 \mathrm{~m}$ DEM for terrain correction. Landsat TM and ETM+ images are then atmospherically corrected using the LEDAPS algorithms described above. The common area for each data cube is then clipped.

The dense time series of images for each data cube pose a major challenge to most existing bi-temporal change detection techniques. To effectively exploit the value of the data cubes for forest disturbance analysis, we have developed a highly automated multi-temporal change analysis method which simultaneously analyzes all images of a data cube. This method first automatically identifies core forest pixels within each image. It then calculates a "forestness" index based on the identified core forest pixels to evaluate the likelihood of each pixel being a forest pixel. Forest disturbances and recovery processes are detected using the temporal profile of the forestness index.

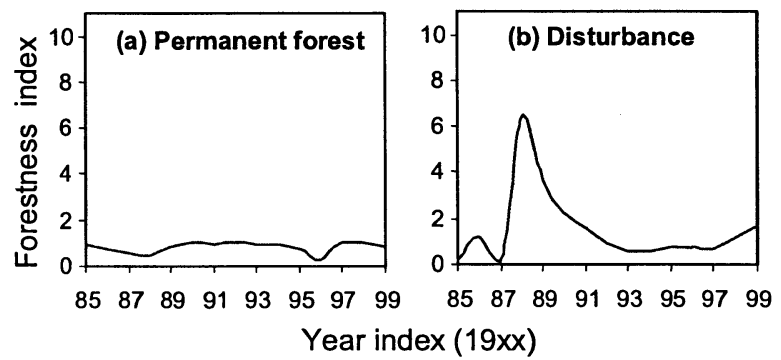

Figure 2. Forestness index through time, showing stable mature forest (left) and the response to a 1988 harvest and subsequent recovery.

An example is shown in Fig. 2, which illustrates the temporal trajectory of the forestness index for a mature stand and a stand disturbed in 1988. The magnitude of the departure of the forestness index gives some indication of the severity of the disturbance, allowing (for example) thinning and clear cuts to be separated. This analysis is carried out for each data cube. Applying this technique to an entire data cube results in maps showing the year of last disturbance, a proxy for stand age (Fig. 3).

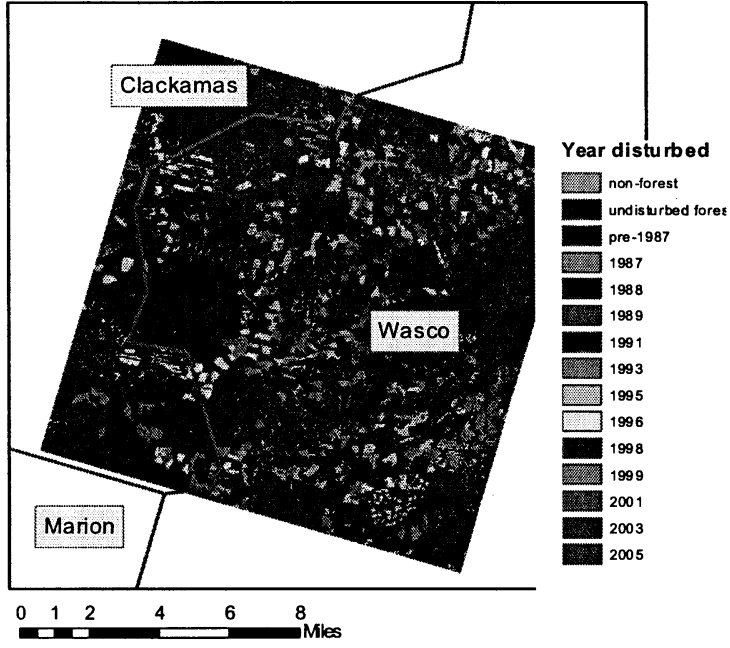

Figure 3. Year of last disturbance for a three-county region of Oregon, 1987-2005, derived from the forestness index algorithm.

\section{Biomass Analysis and FIA Comparisons}

A key component of this study is to understand the dynamics of forest disturbance and regrowth processes as they relate to biomass and carbon dynamics across a range of 
spatial and temporal scales. We developed empirical models to integrate co-located FIA plot-level measurements of forest biomass with Landsat observations of spectral reflectance, to predict live aboveground tree biomass dynamics for each of the Landsat data cubes. The approach is empirical, and hinges upon the direct comparison of Landsat spectral data with plot level observations at co-located FIA plots.

To test this approach, we have assembled FIA plot-level observations of biomass, and ancillary climatic and biophysical predictor variables, for three sample data cube locations. We compared 5 statistical modeling techniques for predicting biomass from spectral and ancillary variables: reduced major axis (RMA) regression, generalized additive models (GAMs), gradient nearest neighbor (GNN) imputation, stochastic gradient boosting (SGB), and Cubist regression trees. For each sample scene, biomass response surfaces for each method were mapped across the population of forested pixels, and validated against independent data. To predict biomass at multiple points in time, the static models were applied to each Landsat date in the data cube, following radiometric cross-normalization, to produce per-pixel estimates of biomass change through time (Fig. 4).

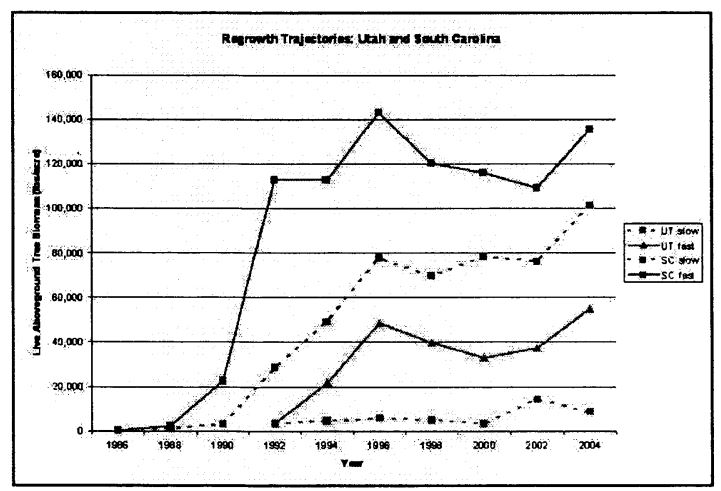

Figure 4. Example biomass accumulation trajectories following harvest derived from Landsat reflectance data from two sites: Utah (red) and South Carolina (blue). "Fast" (solid) and "Slow"(dashed) trajectories are shown for each case, but as expected biomass accumulation in the South Carolina pine ecosystem is much higher than in the semi-arid Utah ecosystem.

The preliminary results from the three sample data cubes suggest that the regression tree methods (SGB and Cubist) are the most accurate in terms of prediction error, and are relatively easy to implement and interpret across a large number of samples.

\section{CONCLUSIONS}

Both the LEDAPS and NACP-FIA projects are providing unique and complementary information on the state of North American forests. The wall-to-wall LEDAPS analysis will allow an improved spatial interpolation of the NACP-FIA sampling results. Conversely, the more temporally accurate NACP-FIA rates will allow the LEDAPS maps to be calibrated. Although disturbance has long been recognized as a fundamental aspect of ecosystem health, these projects represent the first attempts to mine the historical satellite record to characterize disturbance at a continental scale.

This continental mapping allows stand age and turnover to be parameterized within biogeochemical models such as CASA [8]. Since carbon fluxes depend strongly on the distribution of stand ages across the landscape, the LEDAPS products should help to refine estimates of continental ecosystem productivity and its variability.

In the future, we expect that annual, wall-to-wall assessments of forest dynamics would merge the best aspects of both projects. Such an effort would allow accurate estimates of disturbance rates due to "ephemeral" processes such as insect defoliation and storm damage, which are difficult to detect even with one image acquisition every two years. In time, this could transition to a near real-time forest monitoring capability. Processing an annual, wall-to-wall Landsat data set for North America is no longer beyond available computational capabilities, but it requires open, low-cost access to the historical Landsat archive and an ongoing commitment from funding agencies to support such work.

\section{REFERENCES}

[1] Pacala SW, Hurtt GC, Baker D, et al., "Consistent land- and atmosphere-based US carbon sink estimates", Science, 292, 2316 2320, 2001.

[2] Houghton RA, "The annual net flux of carbon to the atmosphere from changes in land use 1850-1990", Tellus Series B-Chemical And Physical Meteorology, 51, 298-313, 1999.

[3] Thornton PE, Law BE, Gholz HL, Clark KL, Falge E, Ellsworth DS, Golstein AH, Monson RK, Hollinger D, Falk M, Chen J, Sparks JP., "Modeling and measuring the effects of disturbance history and climate on carbon and water budgets in evergreeen needleleaf forests", Agricultural and Forest Meteorology 113, 185-222, 2002.

[4] Tucker, CJ., D.M. Grant, and J.D. Dykstra, "NASA's global orthorectified Landsat data set", Photogrammetric Engineering and Remote Sensing, 70, 313-322, 2004.

[5] Vermote, E.F Vermote EF, El Saleous N, Justice CO, Kaufman YJ, Privette JL, Remer L, Roger JC, Tanre D, “Atmospheric correction of visible to middle-infrared EOS-MODIS data over land surfaces: Background, operational algorithm, and validation", J. Geophys. Res. 102, 17131-17141, 1997.

[6] Healey, S.P., Cohen, W.B., Zhiqiang, Y., Krankina, O.N "Comparison of tasseled cap-based Landsat data structures for use in forest disturbance detection", Remote Sensing of the Environment, 97, 301-310, 2005.

[7] Gallego, F.J., "Stratified sampling of satellite images with systematic grid of points," ISPRS J. Photogramm. Remote Sens., 59, 369-376, 2005.

[8] Masek, J. G., and Collatz, G.J., "Estimating forest carbon fluxes in a disturbed southeastern landscape: Integration of remote sensing, forest inventory, and biogeochemical modeling", J. Geophys. Res. 111, G01006, doi:10.1029/2005JG000062, 2006. 